\title{
Prospects for energy harvesting using ferroelectric/ferroelastic switching
}

\author{
Wenbin Kang ${ }^{1}$, John E. Huber ${ }^{1 \mathrm{a}}$ \\ ${ }^{1}$ Department of Engineering Science, University of Oxford, Parks Rd, OX1 3PJ, U.K. \\ ${ }^{\text {a)Corresponding author, Email: john.huber@eng.ox.ac.uk }}$
}

\begin{abstract}
Piezoelectric transducers have been widely employed for energy harvesting from vibration or kinetic energy sources. These systems, however, suffer from low energy density and consequently low power density at frequencies corresponding to common ambient vibrations. An alternative approach, using ferroelectric and ferroelastic switching offers potentially much greater energy density, at the cost of loss of linearity. Using a simple model of switching, a working cycle that could generate electrical energy from a harmonically varying source of stress is explored. The cycle uses depolarization by stress, followed by repolarization with combined electromechanical loading. A harvesting electric field and bias electric field are imposed to ensure a stable repeatable working cycle during the depolarization process and repolarization process, respectively. The bias electric field affects ferroelectric/ferroelastic switching, leading to a preferred direction of repolarization. By contrast, without bias electric field, stress alone would not trigger repolarization because of mechanically equivalent states with opposite polarization. The results illustrate that the bias electric field can be much lower than the harvesting electric field, requiring only a small electrical energy input during the cycle. Finally, the conversion efficiency of this cycle is estimated and improvements to the cycle are explored by adjusting the electrical and mechanical field amplitudes.
\end{abstract}

Keywords: energy harvesting, vibration, ferroelectric 


\section{Introduction}

Technological development has promoted mobile electronics, biomedical implanted devices, and wireless sensor network systems. However, the limited lifetime and energy density of batteries, and the inflexibility of wired power sources cannot satisfy all of the resulting power requirements [1-4]. For example, some implanted devices should not be replaced frequently; some sensors are remotely located and must operate for long periods without servicing. Therefore, it is reasonable and promising to use energy harvesting technologies to convert energy from ambient vibrations or motion into electricity. This has the further advantage of providing renewable energy [5-9], thereby avoiding the ongoing cost of replacing batteries or installing wired systems. Among all the power sources, vibrations, which can be created by fluid flows, vehicle motion or human motion, are relatively available in a range of different environments. Thus there has been great interest in vibration energy scavenging based on piezoelectrics, electromagnetics and electrostatics [8,9]. Given their simplicity and efficiency, piezoelectric materials have considerable potential for energy harvesting; various kinds of piezoelectric energy harvesters have been proposed and employed in last decade [10-13].

Existing research has shown that piezoelectric energy harvesters perform most effectively and efficiently near resonance [14-16]. Researchers have attempted to improve their efficiency by optimizing their structures with a view to frequency response. While some concentrated on reducing or controlling the resonant frequency to fit the ambient vibration source frequency, others broadened bandwidth to enhance adaptability[3, 5-9, 17-22]. Researchers also explored piezoelectric energy harvesting from multi-axis vibrations, through innovative design of the piezoelectric component and distribution of inertial mass[23, 24]. Further design enhancements improved efficiency and effectiveness through material choice, operating mode $\left(d_{31}\right.$ versus $\left.d_{33}\right)$ and device geometry[10, 16, 25-31].

Ferroelectric materials offer the potential for increased power density, relative to piezoelectrics, by exploiting ferroelectric switching, which is a non-linear phenomenon resulting from the presence of multiple symmetry variants below the Curie temperature [32]. Switching is normally induced by external electric field, but can also be driven by stress, in which case it is known as ferroelastic switching. A few examples of ferroelectric/ferroelastic energy harvesters have been proposed[33-36]. Balakrishna et al. [34] developed a concept for a thin-film ferroelectric energy harvester. When a tetragonal ferroelectric material experiences uniaxial tensile stress, the spontaneous polarization vectors can align with the axis of stress due to 
ferroelastic switching. With appropriately placed electrodes, the process can drive a flow of charge. By contrast, compressive stress can make the spontaneous polarization vectors switch back, reversing the charge flow. The resulting output power density is greater than typical piezoelectric energy harvesters. However, the design proposed by Balakrishna et al. [34] relies on a specific domain pattern that is difficult to fabricate, and may be susceptible to mechanical shock. Wang et al. [35, 36]proposed an improved design, using a bias field to stabilize the energy harvester. The prototypes are similar to existing cantilever-type piezoelectric energy harvesters, with the advantage of simplicity of manufacture.

Building on previous work, a novel energy harvesting method depending on ferroelectric switching is discussed in this paper. The system works by partially depolarizing a polycrystalline ferroelectric material with stress, and then repolarizing the material with combined electromechanical loading. The working cycle does not require any specific microstructure or domain patterning, so it could be used with ordinary bulk ferroelectrics. A possible realization would generate the stresses needed in the ferroelectric component by attaching it to a substrate whose curvature is varied during the harvesting cycle. Thus cantilever devices, similar to existing piezoelectric energy harvesters, could be used. The only added complexity is provided by external control of voltages, so the device would require a more complex external energy harvesting circuit than that needed for a piezoelectric energy harvester. For an initial study, we use Hwang's model of switching [37] to explore potential working cycles. This model has the advantage of simplicity and speed, though more sophisticated models, backed by experimental measurements, would be required for a fuller analysis. From the preliminary results, it is found that a working cycle with positive net energy output can be achieved. Additionally, the conversion efficiency of the cycle is estimated and improved by adjusting the electrical and mechanical field amplitudes.

\section{Concept for a ferroelectric/ferroelastic energy harvester}

Ferroelectric ceramic materials are produced by sintering powders, and when cooled to below the Curie temperature, they adopt a ferroelectric state such as a polar tetragonal, orthorhombic or rhombohedral crystal structure. For simplicity, we consider a tetragonal ferroelectric capable of $90^{\circ}$ and $180^{\circ}$ switching, as investigated by Lynch and Hwang et al. [32, 37] among others. Consider a unit cell of one crystal polarized parallel to the $z$-axis, as shown in figure 1a. We restrict attention to the case where the unit cell has axes $c$ and $a$, with $c>a$. The $180^{\circ}$ switching process can be induced by electric fields in the $-z$ direction that exceed the coercive electric field $E_{c}$ and reverse the polarization direction. In addition to this, uniaxial 
tensile stress along the $x$-axis can cause $90^{\circ}$ switching, but the new direction of polarization is uncertain due to symmetry of the tetragonal unit cell, as shown in figure $1 \mathrm{~b}$. Similarly, in figure $1 \mathrm{c}$, the uniaxial compressive stress can give rise to $90^{\circ}$ switching with an equal probability of four polarization directions. However, when a bias electric field, $E_{\mathrm{B}}$, is applied in the $+z$ direction at the same time as the uniaxial compressive stress, then the $90^{\circ}$ switching has a preferred direction, as shown in figure $1 \mathrm{~d}$. The bias field $E_{\mathrm{B}}$ can be much less than $E_{c}$. A cycle of switching can be made to drive charge onto and off surface electrodes, thereby providing an energy harvesting process in which mechanical energy is converted to electrical energy.

(a)

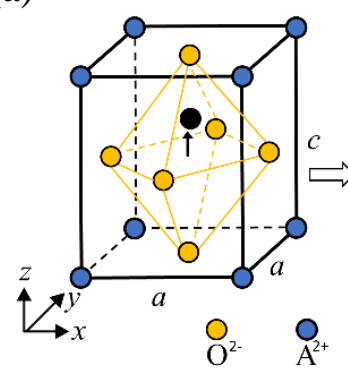

(c)

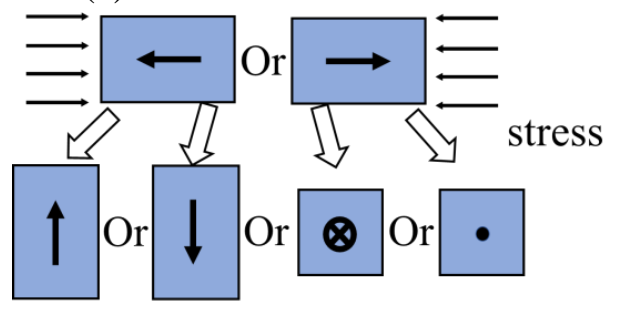

(b)
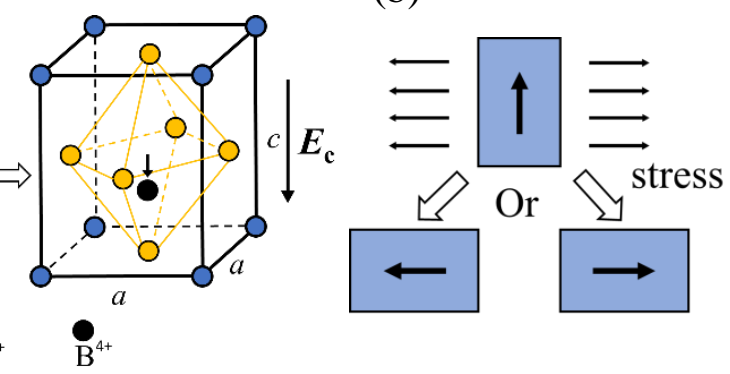

(d)

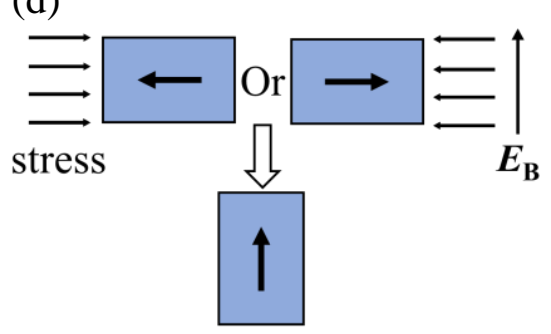

Figure $1.90^{\circ}$ and $180^{\circ}$ ferroelectric/ferroelastic switching processes (a) A tetragonal unit cell with $c>a$. $180^{\circ}$ switching is induced by an electric field (b) Uniaxial tensile stress causes $90^{\circ}$ switching, aligning polarization vectors with an axis. (c) Uniaxial compressive stress causes $90^{\circ}$ switching, aligning polarization vectors with a plane. (d) $90^{\circ}$ switching induced by compressive stress and bias electric field together aligns polarization vectors with a preferred direction.

One way of realizing such a process is illustrated in Figure 2. A thin ferroelectric layer is bonded to a conductive substrate whose curvature then imposes strain on the ferroelectric material. The device is provided with an upper electrode, connected to an external circuit and the substrate is held at zero voltage. In the initial state (figure 2a), the ferroelectric layer is polarized in the downward direction with positive charges on the top electrode. When a tensile stress is applied to the ferroelectric film (figure $2 b$ ) charge flows from the top electrode to the external circuit. A harvesting voltage can be applied, against which the device does work. The electromechanical repolarization process is as follows: The ferroelectric layer is first relaxed (figure 2c). Then, 
compressive stress and a small bias electric field are applied, figure 2d; this repolarizes the device, allowing it to return to the state in figure $2 \mathrm{a}$.

(a)

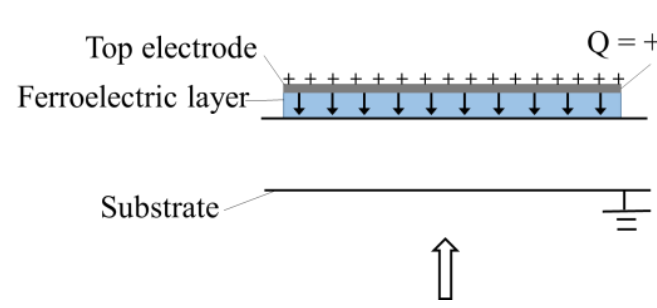

(d)

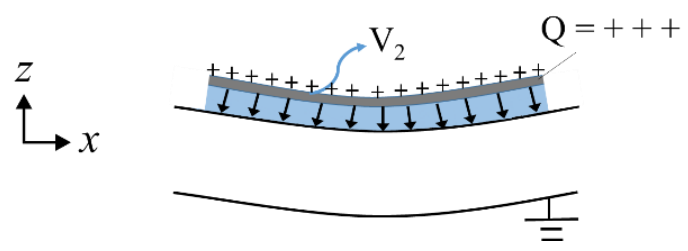

(b)

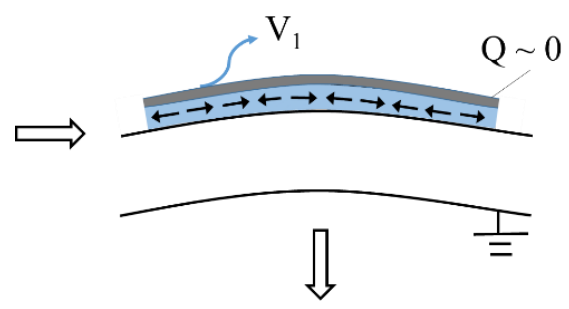

(c)

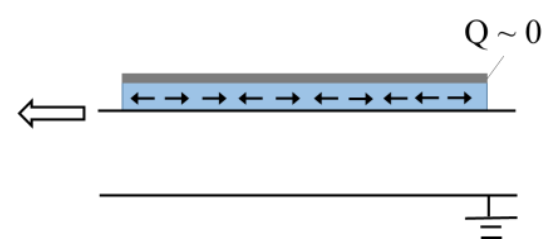

Figure 2. (a)-(d) Schematic illustration of one realization of an energy harvester concept.

While figure 2 illustrates a particular realization of an energy harvesting device, in the present work we explore only the working cycle at a notional material point, making almost no assumptions about the material arrangement, mechanical loading, or external electrical circuit. The only assumptions external to the material model itself are the availability of a harmonically varying, uniaxial stress, aligned with the $x$-axis, and the ability to control electric fields aligned with the $z$-axis. An energy harvesting cycle is then modelled and explored.

The energy harvesting cycle could work with either a single crystal or a polycrystalline ceramic ferroelectric. The macroscopic properties of the ceramic, especially the remanent polarization, arise from a combination of all the individual grains. At the microscopic level, the polarization varies from grain to grain, see figure 3 , and so individual grains will switch to differing degrees. However, the cycle will still work for a macroscopic aggregate of such grains, even though some individual grains may not contribute. The material point modelled here is assumed to consist of a sufficient number of grains such that the behavior of the individual grains is smeared out by the homogenizing effect of the polycrystal. 
(a)

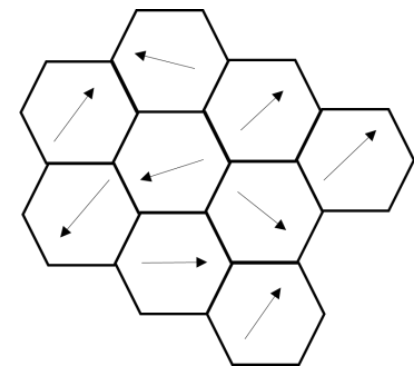

(d) (b)

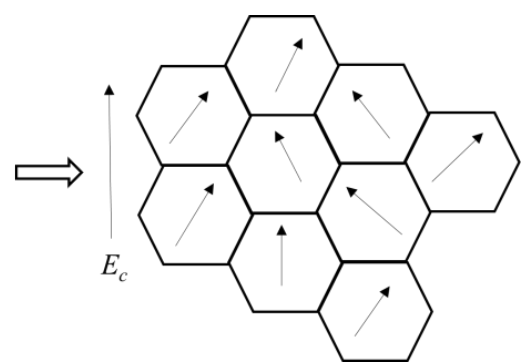

(c)

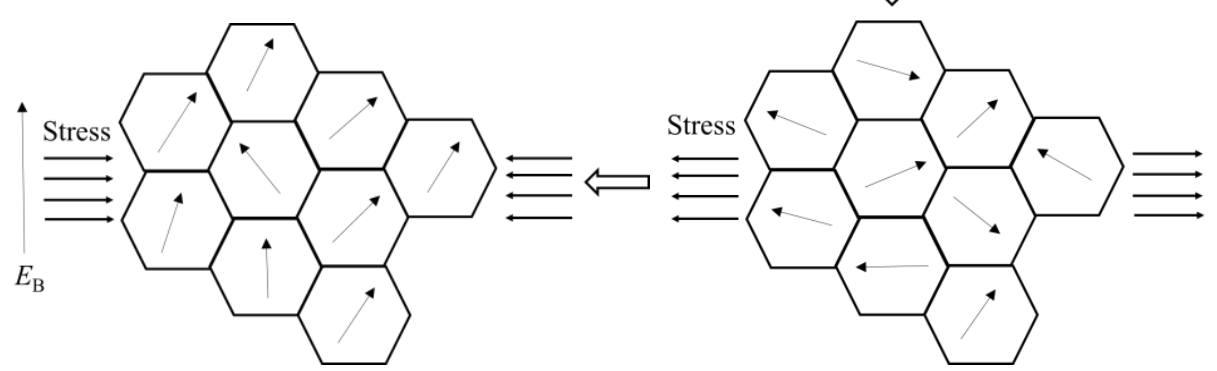

Figure 3. Ferroelectric switching in a ceramic at microscopic level (a) The unpoled state of a ferroelectric ceramic. (b) Switching induced by an electric field above the coercive field. (c) Switching induced by tensile stress above the coercive stress. (d) Switching induced by compressive stress and bias electric field. Hexagonal regions illustrate grains, with average polarization shown by arrows.

\section{Model of the ferroelectric/ferroelastic switching cycle}

The proposed working cycle for energy harvesting is studied by simulating a notional material point, which is a small region of polycrystal consisting of 10000 randomly oriented grains. Note that a typical grain size is $1-5 \mu \mathrm{m}$. Hwang et al. [37] developed a simple ferroelectric/ferroelastic polarization switching model, in order to predict the polarization switching in ferroelectric materials, which has been widely used [38-40]. In the model of Hwang et al. [37], the ceramic is treated as a composite of independently acting grains, with random crystallographic orientations. For simplicity, each grain is regarded as being in a tetragonal state and comprising a single polarized domain. The properties, including polarization and strain of the ceramic, are approximated by the average values over all the grains. Hwang's model does not account for the details of grain-grain interactions and variation of stress or electric field through the ceramic. In this respect it has been superseded by more sophisticated models of switching in ceramics [38]. However, the model has advantages in rapidity, simplicity and reliability. The rapidity of the calculations is particularly advantageous 
for assessing a range of harvesting cycles in order to find and optimize a viable cycle. Hwang's model is also rate independent, and so does not account for the effect of varying cyclic frequency. However, it is known that several material parameters, such as dielectric and piezoelectric properties are frequency dependent[41-43]. Of particular significance, the coercive field and coercive stress can be expected to increase with frequency[44]. Nevertheless, there is evidence that the coercive field is reasonably stable over the likely range of operating frequencies of the energy harvester $(1 \mathrm{~Hz}-1 \mathrm{kHz})[32]$. Mechanical resonance would also be a significant issue in the design of a particular realization of the harvesting cycle.

The strain or remanent polarization in a local coordinate system of each grain can be transformed to a global coordinate system by:

$$
\begin{gathered}
\bar{P}_{j}=a_{k j} P_{k} \\
\overline{e_{i j}}=a_{k i} a_{l j} e_{k l}
\end{gathered}
$$

Where $\bar{P}_{j}$ and $P_{k}$ are the components of the polarization of each grain in the global and local coordinate systems respectively, and the $a_{k j}$ are the direction cosines of each grain's local coordinate system. $\overline{e_{i j}}$ and $e_{k l}$ are the components of the spontaneous strain in the global and local coordinate systems respectively. In the local coordinates, the polarization is aligned to one of the three orthogonal co-ordinate axes and has magnitude $P^{0}$. Similarly, the spontaneous strain components in each grain are $\left(c-a_{0}\right) / a_{0}=\frac{2}{3} e_{0}$ parallel to the polarization direction and $\left(a-a_{0}\right) / a_{0}=-\frac{1}{3} e_{0}$ perpendicular to the direction of polarization, where $e_{0}=(c-a) / a_{0}$ is the axial strain change caused by $90^{\circ}$ switching, while $c, a$ and $a_{0}$ are the edge lengths of the tetragonal unit cell and a reference cubic unit cell respectively. Equal stress and electric field are applied to each grain in the global coordinate system. Each grain is then checked for switching and if a switching criterion is satisfied, its polarization and strain are switched accordingly. Simulation proceeds stepwise applying successive changes in loading and tracking the state of each grain as it switches.

With regard to the switching criterion, Hwang et al.[37] proposed critical values of both electric field and stress for switching, based on the work dissipated during the switching process, and applicable to both $90^{\circ}$ switching and $180^{\circ}$ switching. When the combined electrical and mechanical work for a possible switch exceed the critical value $2 P^{0} E_{0}$ (where $E_{0}$ is equal to coercive electric field), switching occurs. The switching criterion can be expressed by

$$
E_{i} \Delta P_{i}+\sigma_{j k} \Delta e_{j k} \geq 2 P^{0} E_{0}
$$


Where $E_{i}$ and $\sigma_{j k}$ are the components of the applied electric field and stress, and $\Delta P_{i}, \Delta e_{j k}$ are the changes in spontaneous polarization and strain respectively. There can be a possibility for multiple switches to be energetically equivalent, in which case the simulation proceeds by choosing randomly among the equivalent switching processes. By averaging over all the grains in the ceramic, the macroscopic changes in polarization and strain can be estimated. Hence the model provides a very simple way to evaluate the working cycle of an energy harvester concept: externally imposed stresses and electric fields drive the material between states, and the resulting energy flows may be assessed to find whether a net electrical energy output has been achieved. The working mechanism of the energy harvester is ferroelectric/ferroelastic switching but the material need not be fully depolarized or repolarized during the working cycle.

To assess the energy flows in the harvesting cycle, ignoring the reversible linear piezoelectric, dielectric and elastic effects, the net electrical work input over the cycle can be expressed as

$$
W_{\mathrm{e}}=\oint E_{i} d P_{i}
$$

Similarly, the mechanical work input is

$$
W_{\mathrm{m}}=\oint \sigma_{i j} d e_{i j}
$$

The net electrical energy output $-W_{\mathrm{e}}$ can be decomposed into an input part, in which increments $E_{i} d P_{i}$ are positive and an output part, where the work increments are negative, giving

$$
-W_{\mathrm{e}}=W_{\mathrm{e}-\mathrm{out}}-W_{\mathrm{e}-\mathrm{in}}
$$

A similar decomposition for mechanical work allows the various energy flows into and out of the material to be assessed. Whilst several ways of assessing efficiency are possible, in the present work we adopt a simple definition given by

$$
\eta=\frac{-W_{\mathrm{e}}}{W_{\mathrm{m}}}
$$

This measure indicates the conversion efficiency of net mechanical work input into net electrical work output.

\section{Simulation of an energy harvesting cycle}

The simulation is initiated with a random distribution of polarization directions in the grains. The cycle then consists of uniaxial stress applied along the $x$-axis, varying between a maximum tensile stress $\sigma_{\mathrm{T}}$ and a maximum compressive stress $\sigma_{\mathrm{C}}$. At the same time, an electric field is applied parallel to the $z$-axis, with a steady value $E_{\mathrm{H}}$ 
applied while the stress is tensile and increasing in magnitude, followed by zero electric field while the stress is tensile and decreasing. The applied field $E_{\mathrm{H}}$ resists the flow of charge during the depolarizing process; hence the material does external electrical work. During the repolarization process, when the stress is compressive and increasing in magnitude to $\sigma_{\mathrm{C}}$ a steady bias electric field $E_{\mathrm{B}}$ is applied along the $z$-axis, assisting repolarization. The electric field is then reset to zero when the stress is compressive and reducing - see figure 4 .

The cycle is initiated with one of two pre-processes: the first half cycle is either the depolarization process, with tensile stress applied, or the repolarization process, with compressive stress. The first of these cases is shown in figures 4 and 5; here the cycle is initiated by the strong electric field applied concurrently with the tensile stress, and can be thought of as "electric field triggered". The other case, where the repolarization process comes first, is shown in figures 5 and 6 . This case can be thought of as "stress triggered". In either case, the cycle eventually stabilizes, though it may take more than one full cycle to do so.

In figure $4 \mathrm{a}$, the changes of electric field and remanent polarization with time are shown for the electric field triggered process. The results are normalized by the parameters $E_{0}, P^{0}, e_{0}$ and $\sigma_{0}$, where $\sigma_{0}=2 P^{0} E_{0} / e_{0}$. The corresponding changes of stress and remanent strain are shown in figure $4 \mathrm{~b}$, where $\bar{E}_{z}$ and $\bar{P}_{z}^{r}$ are the macroscopic electric field and remanent polarization respectively. $\bar{\sigma}$ and $\bar{e}_{x}^{r}$ are the applied stress and remanent strain in the $x$-axis respectively, and $t / t_{0}$ is the normalized time in the process. Since the model is rate independent, the time scale is arbitrary. The ceramic is initially unpoled with zero remanent polarization. When electric field is applied along the $z$-axis, the ferroelectric material becomes polarized, resulting in a significant increase of the remanent polarization $\bar{P}_{z}^{r}$ and a decrease of the remanent strain $\bar{e}_{x}^{r}$. The electric field is held constant during the energy harvesting stage $\left(0<t<t_{0}\right)$ while tensile stress is applied. During this process, the ceramic only partially depolarizes, because the harvesting electric field prevents the stress driven switching in some grains. Next, the harvesting electric field drops to zero while the stress is unloaded, $t_{0}<t<2 t_{0}$. Following this, the compressive stress and bias electric field are applied in order to repolarize the ceramic. The simulation illustrates that remanent polarization $\bar{P}_{z}^{r}$ increases and remanent strain $\bar{e}_{x}^{r}$ decreases during this stage $\left(2 t_{0}<t<3 t_{0}\right)$. Finally, from $3 t_{0}$ to $4 t_{0}$, the bias electric field returns to zero while the compressive stress is released. The loading cycle is repeated and the material response stabilizes during the second cycle. In the example shown in figures 4 and 5 , the harvesting electric field $E_{\mathrm{H}}=4 E_{0}$ and the bias electric 
field is $E_{\mathrm{B}}=0.2 E_{0}$. The maximum compressive stress was $\sigma_{\mathrm{C}}=6 \sigma_{0}$ and the maximum tensile stress was $\sigma_{\mathrm{T}}=4 \sigma_{0}$. This tensile stress value should be kept low enough to avoid fracture in the ceramic.

(a)

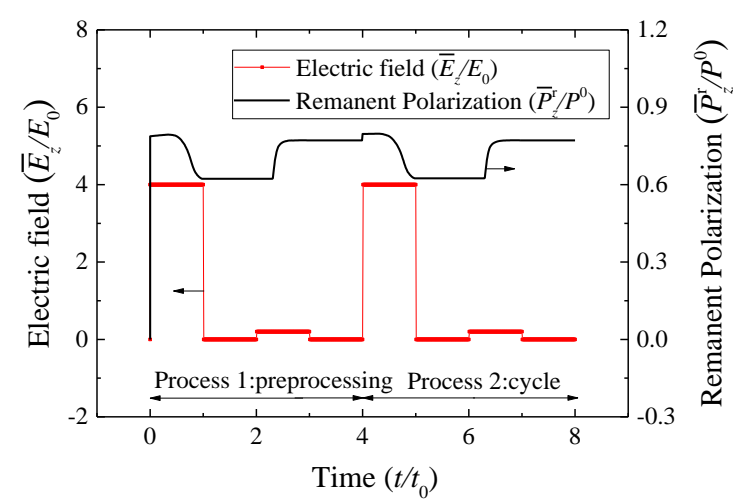

(b)

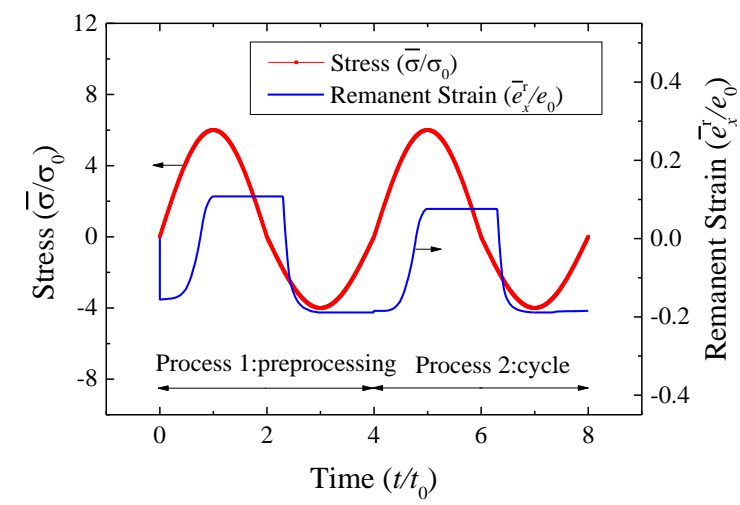

Figure 4. Simulation of the electric field triggered working cycle: (a) electric field and remanent polarization in preprocessing and stable cycle vs time. (b) stress and remanent strain in versus time.

(a)

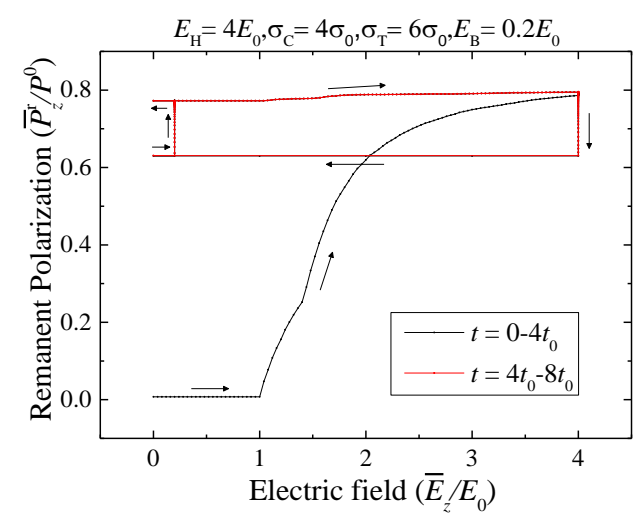

(b)

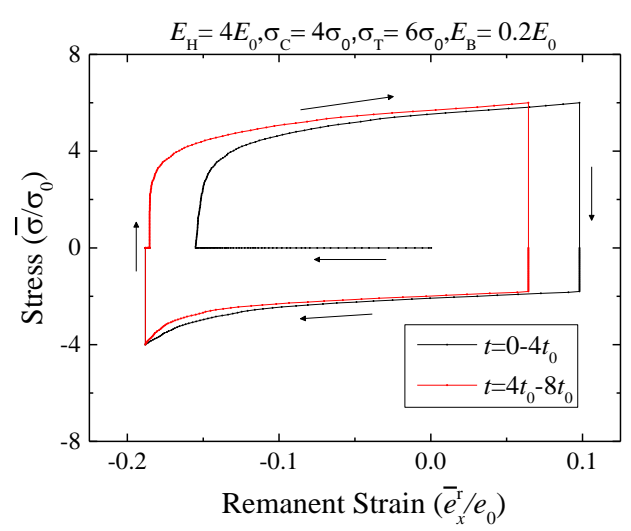

Figure 5. Simulation of the electric field triggered working cycle: (a) remanent polarization versus electric field. (b) stress versus remanent strain.

In figure 5a, the pre-process and stable cycle is shown in electric field - remanent polarization space. Figure $5 \mathrm{~b}$ shows the corresponding change of remanent strain with applied stress in the first two cycles. Initially, the strong electric field causes a rapid polarization process, which produces a negative strain along the $x$-axis. By time $t=t_{0}$, a tensile stress has been applied simultaneously with the high electric field, and the remanent strain has increased non-linearly while a partial depolarization occurred. The tensile stress peaks at approximately $6 \sigma_{0}$ and reduces to zero, after which compressive stress is applied with bias field, causing repolarization. The hysteresis loop closes during the second cycle. 
A second way of initiating the stable harvesting cycle is shown in Figure 6. The parameters used in this case are the same as for the first method, but the cycle starts with compressive stress. During the first stage, $0<t<t_{0}$, although there is a bias electric field inducing polarization, the remanent polarization of the ceramic does not reach the fully polarized state. From $2 t_{0}$ to $3 t_{0}$, the harvesting electric field is applied, which polarizes the ceramic and this initiates the stable energy harvesting cycle.

(a)

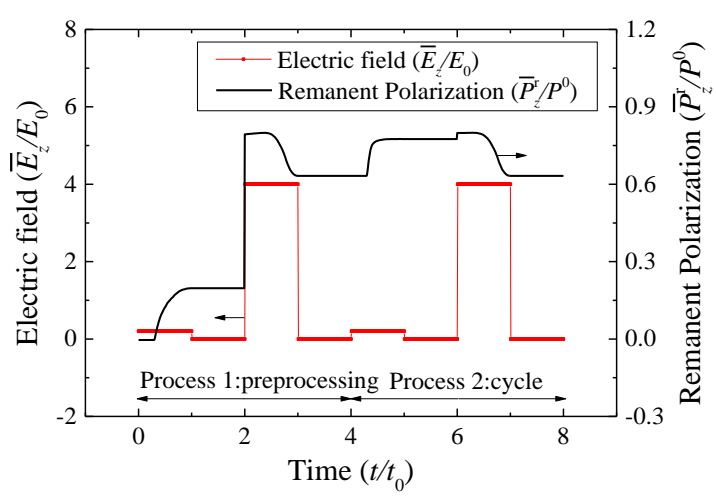

(b)

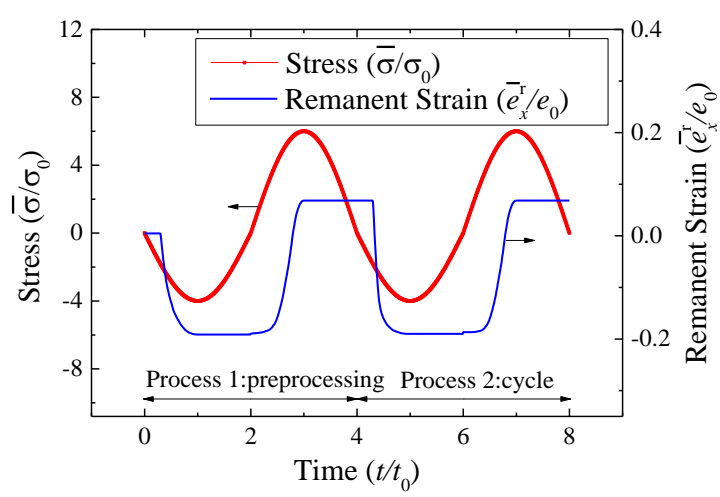

Figure 6. Simulation of bias electric field and compressive stress triggered working cycle: (a) electric field and remanent polarization in preprocessing and stable cycle versus time. (b) stress and remanent strain versus time.

(a)

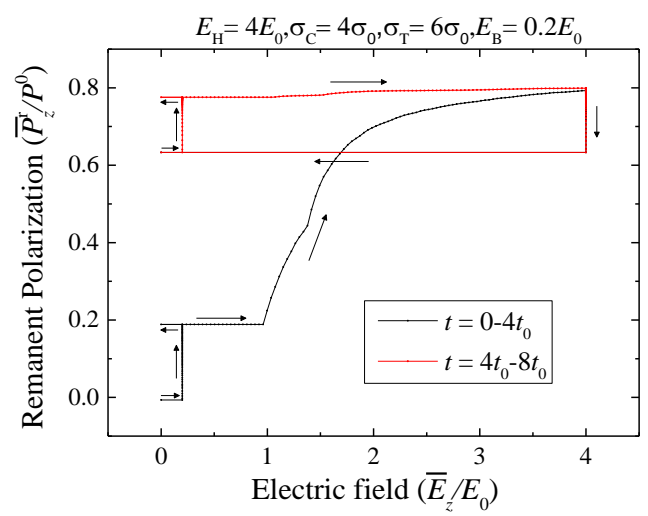

(b)

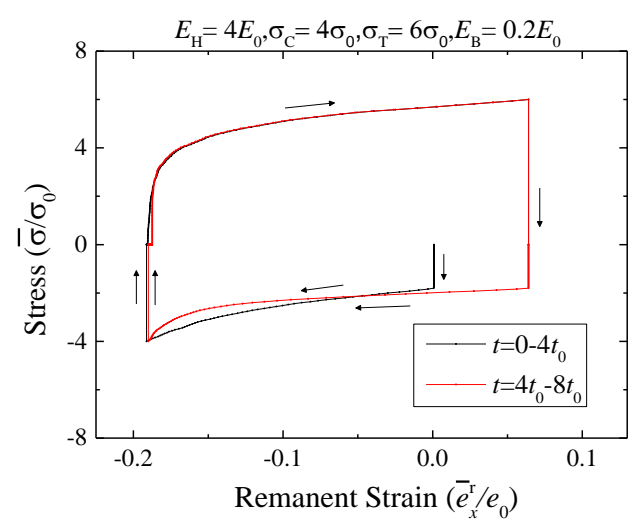

Figure 7. Simulation of working preprocessing of the compressive stress triggered cycle. (a) remanent polarization versus electric field. (b) stress versus remanent strain 
Figure 7, shows the stress-triggered preprocess and the stable energy harvesting cycle. This indicates that, in order to polarize the ceramic, a strong electric field $E_{z}$ is needed for both trigger methods. However the cycle is robust in the sense that if the ferroelectric material becomes fully depolarized at any point, the application of electric field $E_{z}=E_{\mathrm{H}}$ will repolarize the ceramic and restart the energy harvesting cycle. This robustness relies on the harvesting field $E_{\mathrm{H}}$ being greater than the coercive field of the material.

\section{Exploration of the energy harvesting cycle parameters}

Based on simulations, the conversion efficiency of this energy harvesting cycle can be estimated and the cycle optimized. By varying the values of harvesting electric field $E_{\mathrm{H}}$, bias electric field $E_{\mathrm{B}}$, maximum tensile stress $\sigma_{\mathrm{T}}$, and maximum compressive stress $\sigma_{\mathrm{C}}$, a parametric search for an improved working cycle is made.

First consider the effect of varying the harvesting electric field $E_{\mathrm{H}}$, while keeping $\sigma_{\mathrm{C}}=4 \sigma_{0}, \sigma_{\mathrm{T}}=6 \sigma_{0}$, and $E_{\mathrm{B}}=0.2 E_{0}$. Figure 8 shows a selection of stable remanent polarization-electric field hysteresis loops and the corresponding stress-remanent strain hysteresis. The harvesting field is varied in the range $1-10 E_{0}$. The magnitude of the harvesting electric field should be neither too great, nor too small. If it is too small, there is little electrical work done against the field during the energy harvesting stage, but if it is too great, it will hold the polarization aligned with the $z$-axis, and prevent switching.

(a)

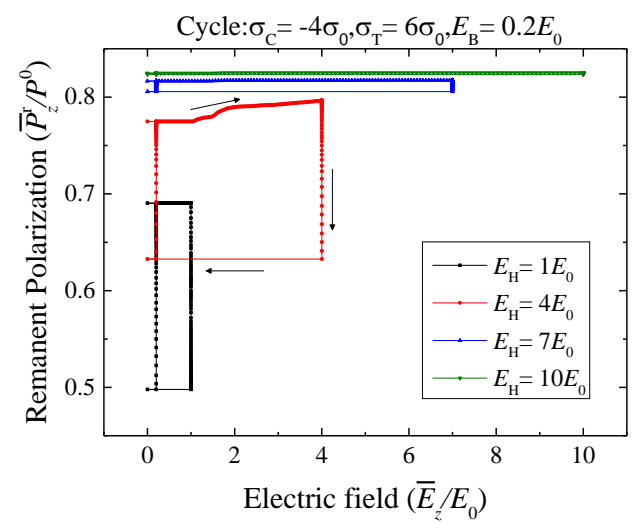

(b)

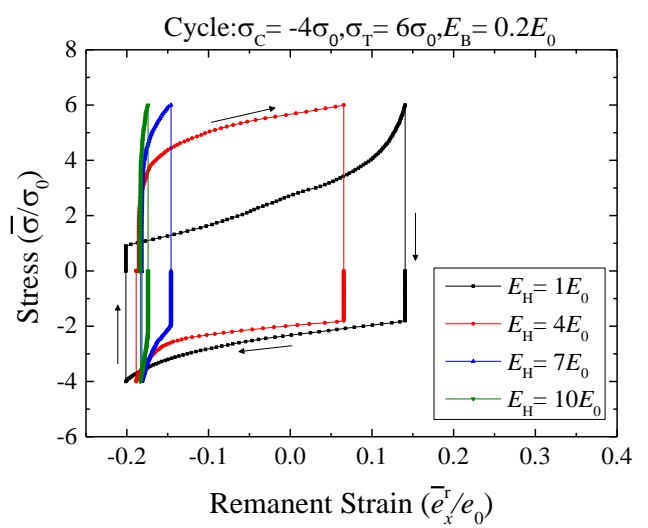

Figure 8. The effect of harvesting electric field $E_{\mathrm{H}}$ on the energy harvesting cycle: (a) Remanent polarization versus electric field. (b) Stress versus remanent strain. 
(a)

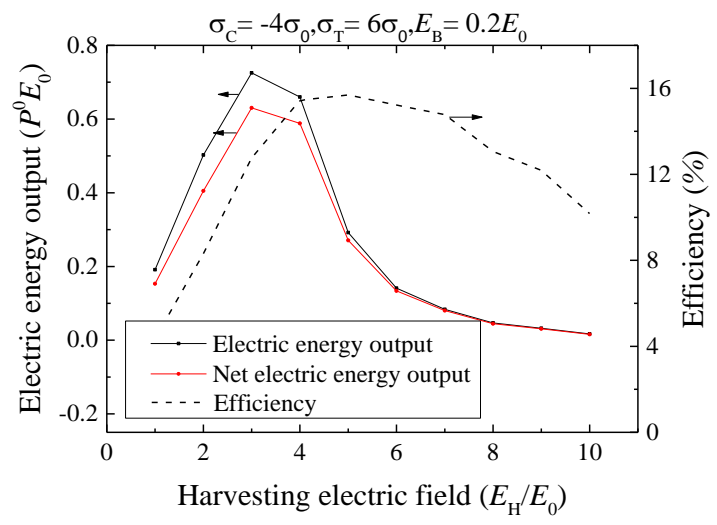

(b)

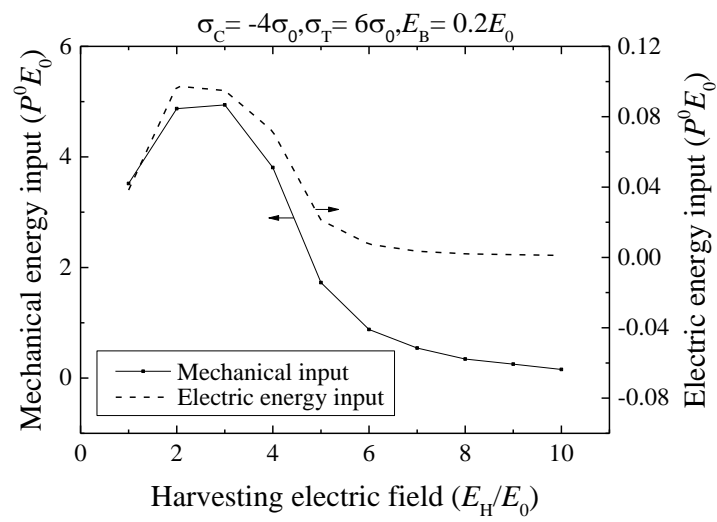

Figure 9. The effect of harvesting electric field $E_{\mathrm{H}}$ on energy flows (a) The electric energy output and conversion efficiency. (b) Energy inputs.

Figure 9a shows the electrical energy output and energy conversion efficiency at various values of harvesting electric field $E_{\mathrm{H}}$. With the increase of harvesting electric field, the electrical energy output and net electrical energy output first rise to their peaks at about $0.75 P^{0} E_{0}$ and $0.65 P^{0} E_{0}$ respectively, and then reduce as $E_{\mathrm{H}}$ is increased beyond $3 E_{0}$. A good choice for $E_{\mathrm{H}}$ appears to be about 3-4 $E_{0}$ in order to maximize energy output. Furthermore, the choice of $E_{\mathrm{H}}$ strongly affects the conversion efficiency, which reaches a maximum of around $16 \%$, when $E_{\mathrm{H}}=4 E_{0}$.

Figure $9 \mathrm{~b}$ shows how the electrical energy input and mechanical energy input vary with $E_{\mathrm{H}}$. The mechanical energy input peaks at about $5 P^{0} E_{0}$ when $E_{\mathrm{H}}=4 E_{0}$ is applied, while approximately $0.1 P^{0} E_{0}$ of electrical energy is put into the cycle. Thus, the mechanical energy is the main energy source; only a small amount of electrical energy input is required.

Next consider the repolarization process, and variation of the bias electric field $E_{\mathrm{B}}$. Figure 10 shows the electrical and mechanical hysteresis loops for $E_{\mathrm{B}}$ values in the range 0.1-1 $E_{0}$. The other parameters are held at $\sigma_{\mathrm{C}}=4 \sigma_{0}, \sigma_{\mathrm{T}}=6 \sigma_{0}$, and $E_{\mathrm{H}}=4 E_{0}$. The results show that varying the bias electric field does not greatly affect the process. A small magnitude of $E_{\mathrm{B}}$ has the effect that some of the repolarization is driven by the harvesting field $E_{\mathrm{H}}$ instead of the intended stress-driven repolarization process. Thus as $E_{\mathrm{B}}$ is reduced, the cycle efficiency reduces, see Figure 11. 
(a)

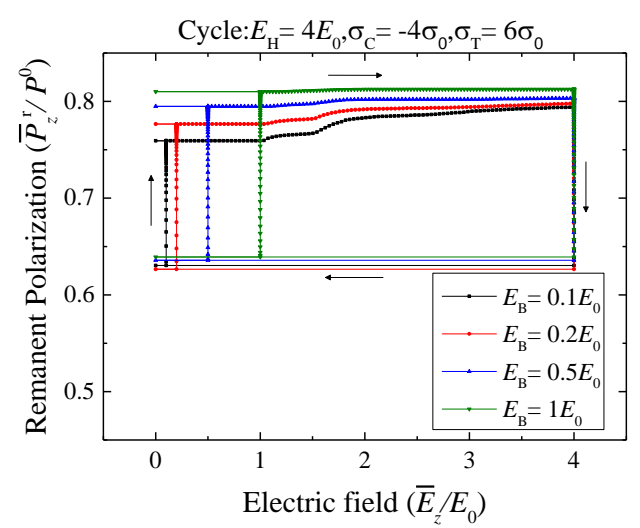

(b)

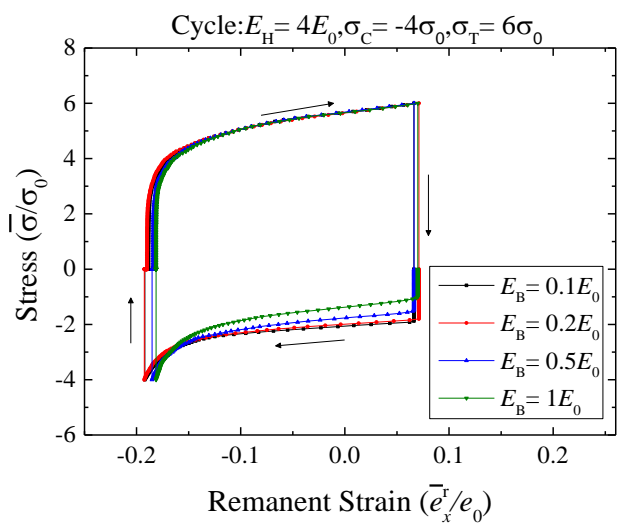

Figure 10. The effect of varying the bias electric field: (a) Remanent polarization versus electric field. (b) Stress versus remanent strain.

(a)

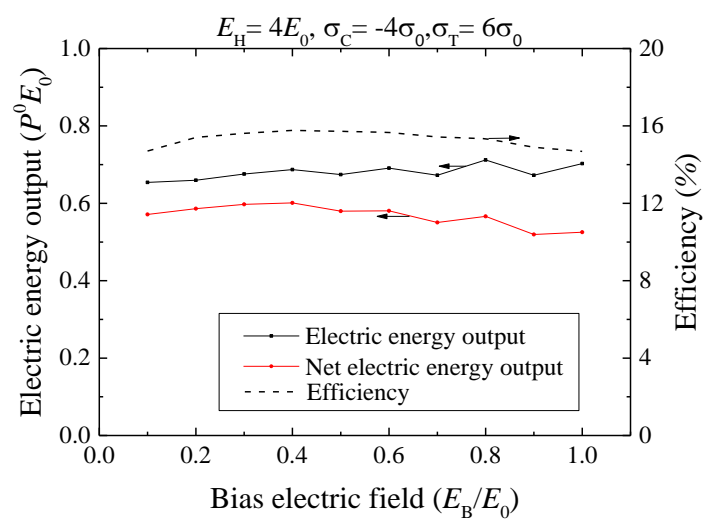

(b)

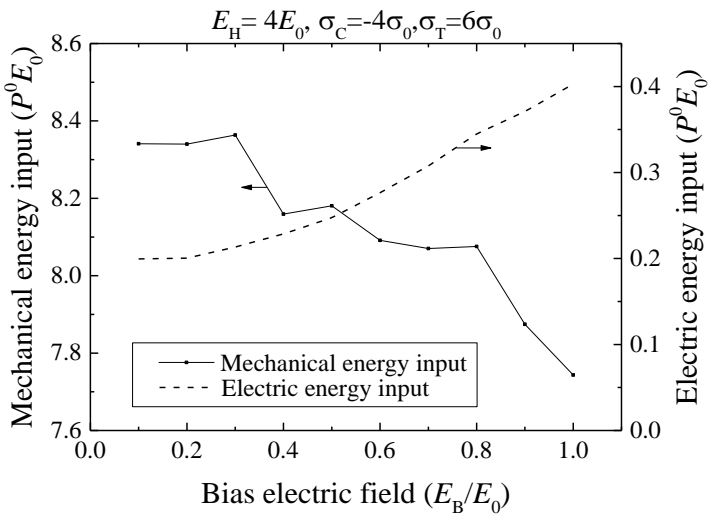

Figure 11. The effect of varying the bias electric field: (a) Electrical energy output and conversion efficiency (b) Energy inputs.

The maximum compressive stress, $\sigma_{\mathrm{C}}$, can also affect the repolarization process. Figure $12 \mathrm{a}$ shows the effect of varying $\sigma_{\mathrm{C}}$ in the range $3-6 \sigma_{0}$, keeping $E_{\mathrm{B}}=0.2 E_{0}$, $\sigma_{\mathrm{T}}=6 \sigma_{0}$ and $E_{\mathrm{H}}=4 E_{0}$. Counterintuitively, increasing the maximum compressive stress does not necessarily enhance the repolarization process. Note that uniaxial compression along the $x$-axis tends to align the polarization vectors with the $y$ - $z$ plane. Thus, for high values of $\sigma_{\mathrm{C}}$, some grains with polarization direction close to the $z$-axis can undergo ferroelastic switching to states that force the polarization away from the $z$-axis. The result is a reduction in polarization. The $z$-axis polarization, $\bar{P}_{z}^{r}$, as a function of $x$-axis uniaxial compressive stress $\sigma$ is shown in figure $12 \mathrm{~b}$, with various values of bias field $E_{\mathrm{B}}$. The reduction in $\bar{P}_{z}^{r}$ at high values of $x$-axis compressive stress can be seen. Notice also that, when $E_{\mathrm{B}}=0$, the compressive stress tends to depolarize the ceramic. A small magnitude of bias field, $E_{\mathrm{B}}=0.2 E_{0}$ is sufficient to allow repolarization by compressive stress, and stronger bias field 
generally enhances the repolarization process. The reduction in efficiency with increasing magnitude of compressive stress can be understood with reference to figure 13(b). Increasing the compressive stress magnitude also increases the mechanical work input. However, as can be seen from figure 12(a), the electrical work output does not change much. Hence the efficiency falls off with increasing magnitude of $\sigma_{\mathrm{C}}$. Figure $12 \mathrm{c}$ shows the remanent strain versus stress hysteresis loops, corresponding to the cases shown in figure $12 \mathrm{a}$.

(a)

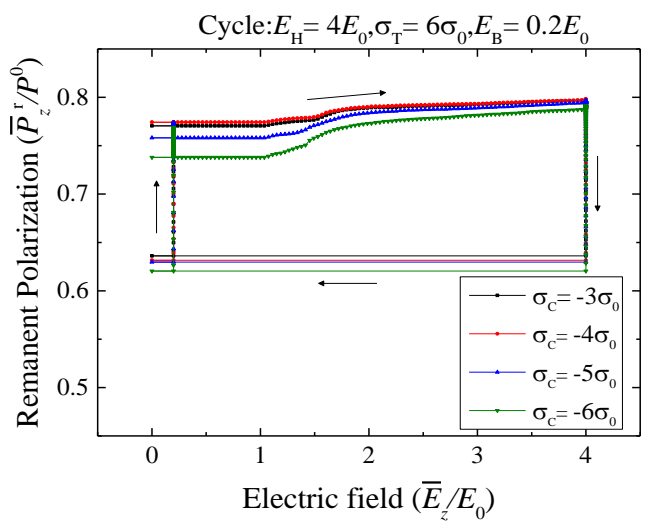

(b)

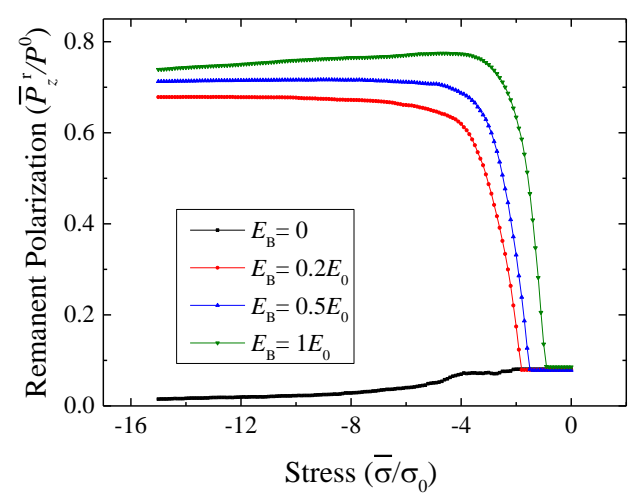

(c)

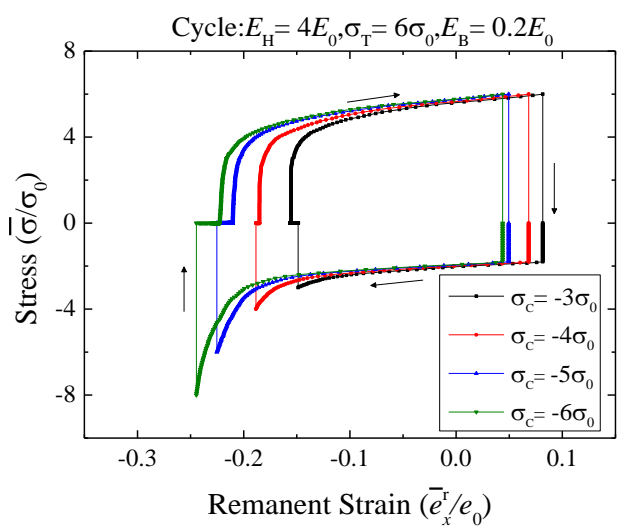

Figure 12. The effect of maximum compressive stress on the energy harvesting cycle (a) Remanent polarization versus electric field. (b) The repolarization process at various values of bias field (c) Stress versus remanent strain. 
(a)

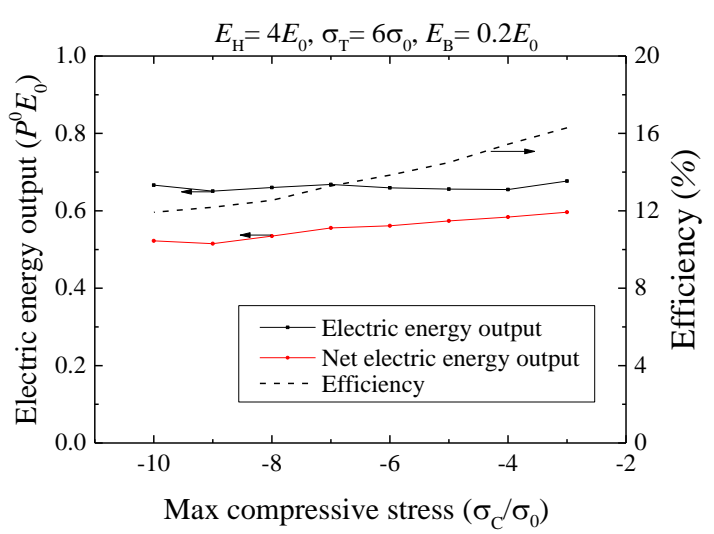

(b)

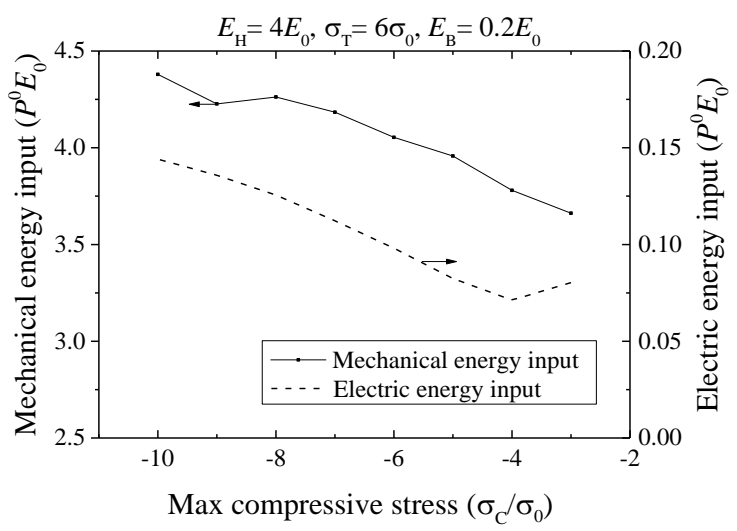

Figure 13. . The effect of maximum compressive stress on the energy harvesting cycle (a) Electrical energy output and conversion efficiency (b) Energy inputs.

The efficiency and energy flows corresponding to figure 12 are shown in figure 13. Evidently, although varying $\sigma_{\mathrm{C}}$ has some effect on the mechanical and electrical energy inputs, it does not greatly affect the outputs or overall efficiency. The choice of $\sigma_{\mathrm{C}}$ can then be largely based on providing the minimum compression sufficient to substantially repolarize the ceramic; $\sigma_{\mathrm{C}}=4 \sigma_{0}$ appears sufficient. The importance of the bias electric field $E_{\mathrm{B}}$ is most clearly seen in figure 12(b). If $E_{\mathrm{B}}$ is too small, the repolarization process does not happen and the cycle breaks down. However, provided $E_{\mathrm{B}}$ is great enough to enable the cycle, it has relatively little effect on energy output or efficiency-see figure 11(a).

Finally, the influence of the maximum tensile stress along the $x$-axis, $\sigma_{\mathrm{T}}$, is explored. Due to the risk of fracture, the maximum tensile stress magnitude should generally be kept small. Initial estimates based on data for PLZT [45] suggest that $\sigma_{\mathrm{T}}<10 \sigma_{0}$ may be acceptable. However, specific tests in the device geometry would be needed to confirm safe tensile loading limits. Figure 14 shows the effect of varying $\sigma_{\mathrm{T}}$ on the harvesting cycle, keeping other parameters constant as before. This shows the importance of the tensile stress for depolarizing the ceramic: with $\sigma_{\mathrm{T}} \leq 3 \sigma_{0}$ almost no depolarization is seen. Increasing $\sigma_{\mathrm{T}}$ causes more grains to switch into polarization directions aligned to the $\pm x$ axis, and so decreases the remanent polarization $\bar{P}_{z}^{r}$ of the ceramic. Thus, greater tensile stress is desirable to improve the working cycle of the energy harvester. When $\sigma_{\mathrm{T}} \geq 6 \sigma_{0}$, the enlarged stress-remanent strain loop shows an effective working cycle. Figure 15 shows that efficiency and electrical work output increase with $\sigma_{\mathrm{T}}$, but the efficiency does not increase much beyond $\sigma_{\mathrm{T}}=6 \sigma_{0}$ due to the gradual exhaustion of switching systems in the depolarization process. The analysis suggests that $\sigma_{\mathrm{T}}$ should be at least $6 \sigma_{0}$ 
and as great as possible whilst avoiding mechanical failure.

(a)

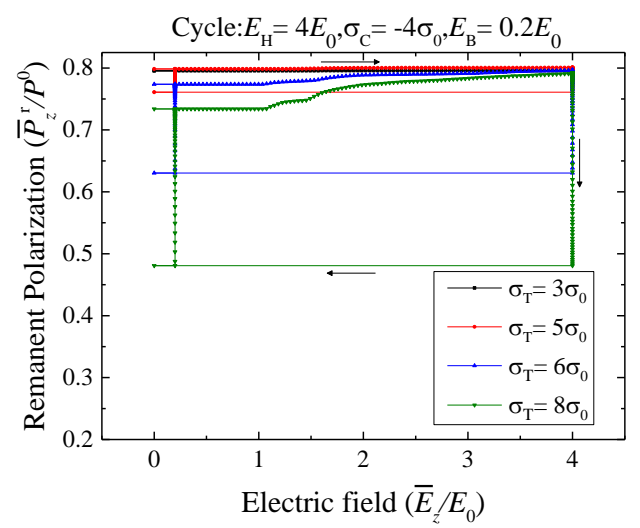

(b)

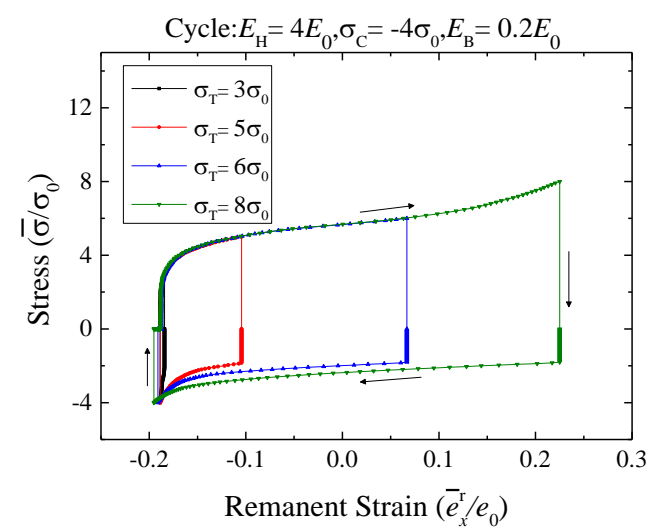

Figure 14. The effect of varying the maximum tensile stress: (a) Remanent polarization versus electric field. (b) stress versus remanent strain.

(a)

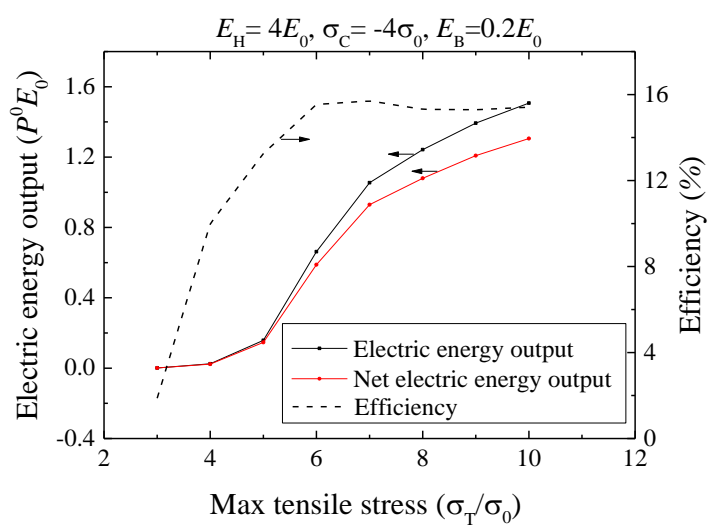

(b)

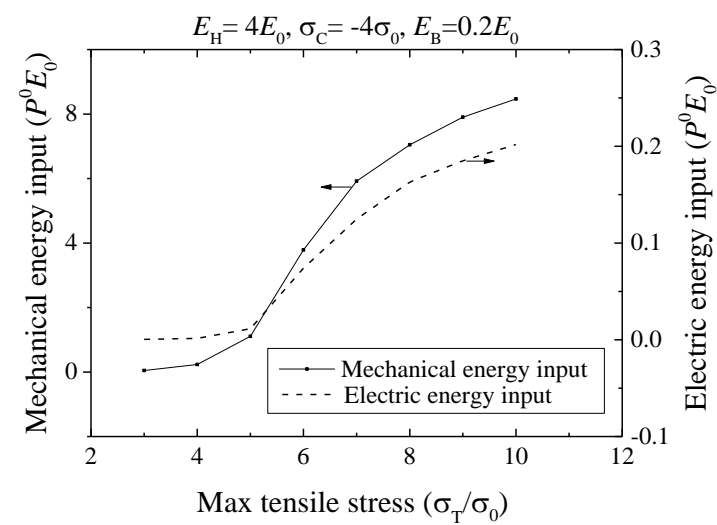

Figure 15. The effect of varying the maximum tensile stress on the energy harvesting cycle: (a) Electrical energy output and conversion efficiency (b) Energy inputs.

The performance of the proposed energy harvesting cycle can be assessed in comparison to other proposed or real devices. For instance, based on the above analysis, an $8 / 65 / 35$ PLZT layer with $P^{0}=0.3 \mathrm{Cm}^{-2}$ and $E_{0}=0.36 \mathrm{MVm}^{-1}$ would generate about $0.16 \mathrm{Jm}^{-2}$ per micrometre of thickness on each cycle and offer area power density of $160 \mathrm{Wm}^{-2}$ per micrometre of thickness at $1 \mathrm{kHz}$ under ideal conditions (neglecting losses in the external circuit). This power density is much greater than that of existing piezoelectric energy harvesters, and several times greater that of the proposed ferroelectric energy harvester concept in reference [34] operating at the same frequency. The conversion efficiency can reach about $16 \% \sim 20 \%$, which is comparable to that of commercial photovoltaics [46-48]. The reported range of 
efficiencies for linear piezoelectric energy harvesters is wide, but values in the region of 10-20\% appear typical[49], suggesting that the proposed cycle may be competitive, in energetic terms, if it can be realized in practice. However, it should be noted that the model used was simplistic, neglecting grain-grain interactions, rate effects, thermal effects and cyclic fatigue in the ceramic, as well as assuming uniformity of stress and strain, that would not be accurate in cantilever-type devices. The theoretical development also neglects several practical difficulties that would need to be considered in developing a working device: fracture and/or mechanical fatigue will limit the acceptable levels of tensile stress, while the electric fields required in some ceramics could exceed the breakdown strength of air, requiring additional insulating layers that may influence mechanical performance. Edge and end effects have been ignored. Similarly, clamping of the ceramic by electrode layers or substrates has been ignored. In particular, clamping of the $y$-axis strain by a substrate could be significant if the widely-used cantilever configuration is employed. The development of an external circuit to control the harvester is also a significant consideration, since an active circuit is required to provide the bias field at the appropriate point in the cycle. Thus further development and experimental confirmation would be needed to be confident of the system's practical value. In this sense, the results reported here represent only an initial assessment of a concept, intended to demonstrate its potential. We are currently developing experimental validation of this energy harvesting concept, to be reported in future work.

\section{Conclusions}

In conclusion, a novel energy harvesting cycle based on ferroelectric switching has been proposed and investigated using Hwang's model of ferroelectric switching. The cycle has the advantage of exploiting large changes in electrical polarization during switching to provide a greater energy density than that usually achieved in piezoelectric energy harvesters. A further advantage is that the cycle could potentially be realized in a simple device such as a thin layer of ferroelectric material on a bending beam substrate: the complex switching cycle is achieved by external electrical control. The energy harvesting cycle was explored by adjusting the electromechanical parameters, and the energy output and conversion efficiency were estimated. The results indicate that the energy harvesting cycle could generate power density and conversion efficiency that are competitive with, or improve upon, existing piezoelectric energy harvesters. This makes the proposed ferroelectric-ferroelastic energy harvesting cycle a promising candidate for further development. 


\section{References}

[1] Cook-Chennault K, Thambi N, Sastry A. Powering MEMS portable devices-a review of non-regenerative and regenerative power supply systems with special emphasis on piezoelectric energy harvesting systems. Smart Materials and Structures. 2008;17:043001.

[2] Cottone F, Gammaitoni L, Vocca H, Ferrari M, Ferrari V. Piezoelectric buckled beams for random vibration energy harvesting. Smart Materials and Structures. 2012;21:035021.

[3] Kim S-G, Priya S, Kanno I. Piezoelectric MEMS for energy harvesting. MRS bulletin. 2012;37:1039-50.

[4] Qiu Y, Zhang H, Hu L, Yang D, Wang L, Wang B, et al. Flexible piezoelectric nanogenerators based on ZnO nanorods grown on common paper substrates. Nanoscale. 2012;4:6568-73.

[5] Balestra F. Beyond CMOS Nanodevices 1: John Wiley \& Sons; 2014.

[6] Heung Soo Kim J-HKaJK. A Review of Piezoelectric Energy Harvesting Based on Vibration. INTERNATIONAL JOURNAL OF PRECISION ENGINEERING AND MANUFACTURING. 2013;12:1129-41.

[7] Kim HS, Kim J-H, Kim J. A review of piezoelectric energy harvesting based on vibration. International journal of precision engineering and manufacturing. 2011;12:1129-41.

[8] Wei C, Jing X. A comprehensive review on vibration energy harvesting: Modelling and realization. Renewable and Sustainable Energy Reviews. 2017;74:1-18.

[9] Yildirim T, Ghayesh MH, Li W, Alici G. A review on performance enhancement techniques for ambient vibration energy harvesters. Renewable and Sustainable Energy Reviews. 2017;71:435-49.

[10] Yu H, Zhou J, Deng L, Wen Z. A vibration-based MEMS piezoelectric energy harvester and power conditioning circuit. Sensors. 2014;14:3323-41.

[11] Zhao J, You Z. A shoe-embedded piezoelectric energy harvester for wearable sensors. Sensors. 2014;14:12497-510.

[12] Leadenham S, Erturk A. Nonlinear M-shaped broadband piezoelectric energy harvester for very low base accelerations: primary and secondary resonances. Smart Materials and Structures. 2015;24:055021.

[13] Guan M, Liao W-H. Design and analysis of a piezoelectric energy harvester for rotational motion system. Energy Conversion and Management. 2016;111:239-44.

[14] Fang H-B, Liu J-Q, Xu Z-Y, Dong L, Wang L, Chen D, et al. Fabrication and performance of MEMS-based piezoelectric power generator for vibration energy harvesting. Microelectronics Journal. 2006;37:1280-4.

[15] Leland ES, Wright PK. Resonance tuning of piezoelectric vibration energy scavenging generators using compressive axial preload. Smart Materials and Structures. 2006;15:1413.

[16] Liu J-Q, Fang H-B, Xu Z-Y, Mao X-H, Shen X-C, Chen D, et al. A MEMS-based piezoelectric power generator array for vibration energy harvesting. Microelectronics Journal. 2008;39:802-6.

[17] Lu F, Lee H, Lim S. Modeling and analysis of micro piezoelectric power generators for micro-electromechanical-systems applications. Smart Materials and Structures. 2003;13:57.

[18] Karami MA, Yardimoglu B, Inman DJ. Coupled out of plane vibrations of spiral beams for micro-scale applications. Journal of Sound and Vibration. 2010;329:5584-99.

[19] Karami MA, Inman DJ. Analytical modeling and experimental verification of the vibrations of the zigzag microstructure for energy harvesting. Journal of Vibration and Acoustics. 2011;133:011002.

[20] Chen Z, Yang Y, Lu Z, Luo Y. Broadband characteristics of vibration energy harvesting using 
one-dimensional phononic piezoelectric cantilever beams. Physica B: Condensed Matter. 2013;410:5-12.

[21] Deng L, Wen Z, Zhao X, Yuan C, Luo G, Mo J. High Voltage Output MEMS Vibration Energy Harvester in $d_{31}$ Mode With PZT Thin Film. Journal of Microelectromechanical Systems. 2014;23:855-61.

[22] Song $M$, Zhang $Y$, Peng $M$, Zhai J. Low frequency wideband nano generators for energy harvesting from natural environment. Nano Energy. 2014;6:66-72.

[23] JX Liu. Analysis of Load Voltage and Output Power for Rainbow Shape Piezoelectric Monomorph Energy Transferring Elements. Acta Aeronautica et Astronautica Sinica. 2011;32.

[24] Park JC, Park JY. Asymmetric PZT bimorph cantilever for multi-dimensional ambient vibration harvesting. Ceramics International. 2013;39:S653-S7.

[25] Roundy S, Leland ES, Baker J, Carleton E, Reilly E, Lai E, et al. Improving power output for vibration-based energy scavengers. IEEE Pervasive computing. 2005;4:28-36.

[26] Ren B, Or SW, Zhang Y, Zhang Q, Li X, Jiao J, et al. Piezoelectric energy harvesting using shear mode $0.71 \mathrm{~Pb}$ (Mg 1/3 Nb 2/3) O 3-0.29 PbTiO 3 single crystal cantilever. Applied Physics Letters. 2010;96:083502.

[27] Wang W, Yang T, Chen X, Yao X. Vibration energy harvesting using a piezoelectric circular diaphragm array. IEEE transactions on ultrasonics, ferroelectrics, and frequency control. 2012;59.

[28] Kim S-B, Park H, Kim S-H, Wikle HC, Park J-H, Kim D-J. Comparison of MEMS PZT cantilevers based on $d_{31}$ and $d_{33}$ modes for vibration energy harvesting. Journal of Microelectromechanical Systems. 2013;22:26-33.

[29] Daqaq MF, Masana R, Erturk A, Quinn DD. On the role of nonlinearities in vibratory energy harvesting: a critical review and discussion. Applied Mechanics Reviews. 2014;66:040801.

[30] Rezaeisaray M, El Gowini M, Sameoto D, Raboud D, Moussa W. Low frequency piezoelectric energy harvesting at multi vibration mode shapes. Sensors and Actuators A: Physical. 2015;228:104-11.

[31] Yang Z, Zu J. Comparison of PZN-PT, PMN-PT single crystals and PZT ceramic for vibration energy harvesting. Energy Conversion and Management. 2016;122:321-9.

[32] Lynch CS. The effect of uniaxial stress on the electro-mechanical response of 8/65/35 PLZT. Acta materialia. 1996;44:4137-48.

[33] Patel S, Chauhan A, Vaish R. A technique for giant mechanical energy harvesting using ferroelectric/antiferroelectric materials. Journal of Applied Physics. 2014;115:084908.

[34] Balakrishna AR, Huber JE. Nanoscale domain patterns and a concept for an energy harvester. Smart Materials and Structures. 2016;25:104001.

[35] Wang D, Wang L, Melnik R. Vibration energy harvesting based on stress-induced polarization switching: a phase field approach. Smart Material Structures. 2017;26.

[36] Wang D, Melnik R, Wang L. Material influence in newly proposed ferroelectric energy harvesters. Journal of Intelligent Material Systems and Structures. 2018:1045389X18783092.

[37] Hwang S, Lynch C, McMeeking R. Ferroelectric/ferroelastic interactions and a polarization switching model. Acta metallurgica et materialia. 1995;43:2073-84.

[38] Hwang SC, Huber JE, McMeeking RM, Fleck NA. The simulation of switching in polycrystalline ferroelectric ceramics. Journal of Applied Physics. 1998;84:1530-40.

[39] Landis CM. Non-linear constitutive modeling of ferroelectrics. Current Opinion in Solid State and Materials Science. 2004;8:59-69. 
[40] Schneider GA. Influence of electric field and mechanical stresses on the fracture of ferroelectrics. Annu Rev Mater Res. 2007;37:491-538.

[41] Kamba S, Bovtun V, Petzelt J, Rychetsky I, Mizaras R, Brilingas A, et al. Dielectric dispersion of the relaxor PLZT ceramics in the frequency range $20 \mathrm{~Hz}-100 \mathrm{THz}$. Journal of Physics: Condensed Matter. 2000;12:497.

[42] Masys A, Ren W, Yang G, Mukherjee B. Piezoelectric strain in lead zirconate titante ceramics as a function of electric field, frequency, and dc bias. Journal of Applied Physics. 2003;94:1155-62.

[43] Thomas R, Mochizuki S, Mihara T, Ishida T. PZT (65/35) and PLZT (8/65/35) thin films by sol-gel process: a comparative study on the structural, microstructural and electrical properties. Thin Solid Films. 2003;443:14-22.

[44] Lente M, Picinin A, Rino J, Eiras J. $90^{\circ}$ domain wall relaxation and frequency dependence of the coercive field in the ferroelectric switching process. Journal of applied physics. 2004;95:2646-53.

[45] Tanimoto T, Yamamoto K, Morii T. Nonlinear stress-strain behavior of piezoelectric ceramics under tensile loading. Applications of Ferroelectrics, 1994 ISAF'94, Proceedings of the Ninth IEEE International Symposium on: IEEE; 1991. p. 394-7.

[46] O'regan B, Grätzel M. A low-cost, high-efficiency solar cell based on dye-sensitized colloidal TiO2 films. Nature. 1991;353:737.

[47] Wang W, Winkler MT, Gunawan O, Gokmen T, Todorov TK, Zhu Y, et al. Device characteristics of CZTSSe thin - film solar cells with 12.6\% efficiency. Advanced Energy Materials. 2014;4.

[48] You J, Dou L, Yoshimura K, Kato T, Ohya K, Moriarty T, et al. A polymer tandem solar cell with $10.6 \%$ power conversion efficiency. Nature communications. 2013;4:1446.

[49] Yang Z, Erturk A, Zu J. On the efficiency of piezoelectric energy harvesters. Extreme Mechanics Letters. 2017;15:26-37. 